Anaesthesist 2021 · 70:873

https://doi.org/10.1007/s00101-021-01033-3

Angenommen: 9. August 2021

Online publiziert: 16. September 2021

(c) Springer Medizin Verlag $\mathrm{GmbH}$, ein Teil von Springer Nature 2021

\section{Leitlinien zur Notfalltherapie der aortoösophagealen Fistel sind erforderlich}

\author{
Julia Johanna Grannemann ${ }^{1}$ - Achim Röper ${ }^{1}$ - Sebastian Rehberg ${ }^{2}$ - Gerrit Jansen ${ }^{2}$ \\ ${ }^{1}$ Klinik für Anästhesiologie, operative Intensivmedizin, Notfallmedizin und Schmerztherapie, \\ Universitätsklinikum OWL, Universität Bielefeld, Campus Klinikum Bielefeld, Bielefeld, Deutschland \\ ${ }^{2}$ Klinik für Anästhesiologie, Intensiv-, Notfall-, Transfusionsmedizin und Schmerztherapie Evangelisches \\ Klinikum Bethel, Universitätsklinikum OWL, Universität Bielefeld, Campus Bielefeld-Bethel, Bielefeld, \\ Deutschland
}

\section{Leserbrief}

Zum Leserbrief von Süss JD (2021) Therapie der aortoösophagealen Fistel unter Berücksichtigung der ESVS-Leitlinie. Anaesthesist. https:// doi.org/10.1007/s00101-021-01032-4

Originalbeitrag

Grannemann JJ, Röper A, Rehberg S et al (2021) Die aortoösophageale Fistel - Eine seltene Differenzialdiagnose der oberen Gastrointestinalblutung. Anaesthesist. https:// doi.org/10.1007/s00101-021-00963-2

Vielen Dank für Ihre sehr interessanten und konstruktiven Anmerkungen zu unserem Artikel „Die aortoösophageale Fistel - Eine seltene Differenzialdiagnose der oberen Gastrointestinalblutung". Sie beziehen sich in Ihrer Anmerkung auf das Kapital 6.3 „Thoracic vascular graft or endograft infection with oesophageal fistula“ in den „European Society for Vascular Surgery (ESVS) (2020) Clinical Practice Guidelines on the Management of Vascular Graft and Endograft Infections", in der das Vorgehen bei Prothese-Infekt-assoziierten aortoösophagealen Fisteln, wie Sie richtig anmerken, ausführlich dargestellt wird [1]. Leider bezieht sich diese Leitlinie nicht auf aortoösophageale Fisteln nach Tumorarrosionen oder nach thorakalen onkologischen Eingriffen. Ebenso fehlen in dieser Leitlinie Empfehlungen bzw. Angaben bezüglich der akutmedizinischen Versorgung unter Zuhilfenahme von Verfahren wie beispielsweise der "resuscitative endovascular balloon occlusion of the aorta" oder der Sengstaken-Sonde. Nichtsdestotrotz stimmen wir mit Ihnen überein, dass diese Leitlinie, ungeachtet der Entität der aortoösophagealen Fistel, eine wichtige Hilfestellung in der Versorgung der betroffenen Patienten bieten kann. Wie Sie richtig anmerken, lag ein wesentlicher Schwerpunkt der Kasuistik auf der Notfallversorgung von Patienten mit AÖF. In diesem Zusammenhang dient die TEVAR ausschließlich als überbrückende Maßnahme bis zur definitiven operativen Sanierung.

Mit freundlichen Grüßen

\section{Korrespondenzadresse}

\section{Julia Johanna Grannemann}

Klinik für Anästhesiologie, operative Intensivmedizin, Notfallmedizin und Schmerztherapie, Universitätsklinikum OWL, Universität Bielefeld, Campus Klinikum Bielefeld

Teutoburger Straße 50, 33604 Bielefeld, Deutschland

julia.johanna.grannemann@gmx.de

Interessenkonflikt. J.J. Grannemann, A. Röper und G. Jansen geben an, dass kein Interessenkonfliktbesteht. S. Rehberg gibt an, medizinischer Berater bei Fresenius Kabi Deutschland zu sein und Honrar- und Reisekostenerstattung von Amomed Pharma, OrionPharma und CLS Behring zu erhalten.

\section{Literatur}

1. Chakfé N, Diener H, Lejay A et al (2020) European Society for Vascular Surgery (ESVS) 2020 Clinical Practice Guidelines on the Management of Vascular Graft and Endograft Infections. Eur J Vasc Endovasc Surg 59(3):339-384. https://doi.org/10. 1016/j.ejvs.2019.10.016. Erratum in: Eur J Vasc Endovasc Surg 60(6):958 